MADPH-97-1030

December, 1997

\title{
Flavor Changing Neutral Currents at $\mu^{+} \mu^{-}$Colliders
}

\author{
L. Reina \\ Physics Department, University of Wisconsin, \\ Madison, WI 53706, USA
}

\begin{abstract}
We illustrate the possibility of observing signals from Flavor Changing Neutral Currents, originating from the scalar sector of a Two Higgs Doublet Model. In particular, we focus on the tree level process $\mu^{+} \mu^{-} \rightarrow$ $\bar{t} c+\bar{c} t$, via scalar exchange in the $s$-channel, as a distinctive process for $\mu^{+} \mu^{-}$colliders.
\end{abstract}

To appear in the Proceedings of the Workshop on Physics at the First Muon Collider and at the Front End of a Muon Collider, Fermilab, November 6-9, 1997. 


\section{Introduction}

The First Muon Collider (FMC) will reach a maximum center of mass energy of $500 \mathrm{GeV}$, exploring all the interesting intermediate regimes. In a second phase, the $\mu^{+} \mu^{-}$collider should upgrade its energy to up to $4 \mathrm{TeV}$ and therefore become a very high energy lepton collider, even compared to NLC.

A high energy lepton collider will be a very promising environment to look for new physics beyond the Standard Model (SM), taking advantage of the large enough energy which will become available at low background rates. As has become common knowledge by now, the great advantage of a $\mu^{+} \mu^{-}$machine will be to allow the study of the $s$-channel production of scalar Higgs like particles, which should occur at a much more conspicuous rate with respect to an $e^{+} e^{-}$ collider $\left(m_{\mu} \simeq 200 m_{e}\right)$.

A very important and direct application of this property will be the study of the Higgs sector of both the SM and its SuperSymmetric (SUSY) extensions, looking for positive or negative evidence of the scalar and pseudoscalar particles which are theoretically predicted in these models. Even if evidence is found at a different machine (for instance a hadron collider), a $\mu^{+} \mu^{-}$machine will offer an optimal energy resolution to sit at resonance and study the properties of these particles, i.e. their masses and their couplings. This subject has been thoroughly covered in some plenary talks [1, 2] and dedicated parallel sessions [3, 4] during this workshop.

Along these lines, we want to discuss here the possibility of further studying the properties of the scalar sector of the SM as well as of many of its extensions, SUSY included, by looking for anomalous Flavor Changing Neutral Currents (FCNC) induced by scalar exchange. It is well known that any extended scalar sector containing identical replicas of the same representation of scalar fields (for instance: $\mathrm{N}$ identical doublets or $\mathrm{N}$ identical pairs of doublets, etc.) can induce FCNC at the tree level. This is due to the possibility of diagonalizing the mass matrix of the fermion fields without diagonalizing each single fermionscalar coupling The interest in models containig many identical generations of scalars (sometimes even in a one-to-one correspondence with the generations of fermions) arises in many string-inspired Grand Unified Models (for instance $\left.E_{6}\right)$ and is therefore of a more general interest.

In order to be more predictive and to limit the number of parameters in our analysis, we focus on a minimal extension of the scalar sector of the SM, a Two Higgs Doublet Model (2HDM). This can be used as a simple model, which we are able to work out to the very last consequences in order to study the compatibility of its predictions with the existing experiments. On a more general ground, our analysis should provide useful hints for a diversity of extensions of the SM, which both theorist and experimentalist are encouraged to study.

\footnotetext{
${ }^{1}$ We assume Yukawa type couplings between fermions and scalars and fermion masses generated through spontaneous symmetry breaking.
} 
Since FCNC are forbidden in the SM, their study can provide us with unambiguous evidence of new physics. However, severe constraints are imposed by the low energy physics of the $K$ - and $B$-mesons, such that FCNC have practically to be avoided in this sector of the theory. This is naturally accomplished by the SM itself via the GIM mechanism, and has to be imposed ad hoc in any $2 \mathrm{HDM}$, by introducing a discrete symmetry [-5], which limits the possible Yukawa couplings between fermions and scalars.

Apart from the experimental constraints coming from $K$ - and $B$-physics, there is no a priori theoretical reason not to have FCNC. Therefore, the assumption of this ad hoc discrete symmetry may be dropped in favor of a more natural one, which takes any Flavor Changing (FC) coupling to a scalar field to be proportional to the mass of the coupled fermions. The basic idea is that a natural hierarcy is provided by the observed fermion masses and this may be transfered to the couplings between fermions and scalar fields [6, 7, 8, 9]. In this way, FCNC are naturally suppressed in the light sector of the theory, while dramatic effects may be seen in processes which involve the heavy quark fields of the third generation, i.e. the top quark.

We illustrate these ideas at work in the following sections, first presenting the model we refer to 10, 11] and then focusing on some FC signals, namely $(\bar{t} c+\bar{c} t)$-production which, if possible at an $e^{+} e^{-}$-collider [12], is even more enhanced and distinctive at a $\mu^{+} \mu^{-}$-collider [13].

\section{The Model}

We explicitly consider in this context only the quark fields, assuming that the discussion of the quark and lepton sectors of the theory can proceed independently. Then, let us consider the quark Yukawa Lagrangian of a 2HDM, which we write as,

$\mathcal{L}_{Y}^{(I I I)}=\eta_{i j}^{U} \bar{Q}_{i, L} \tilde{\phi}_{1} U_{j, R}+\eta_{i j}^{D} \bar{Q}_{i, L} \phi_{1} D_{j, R}+\xi_{i j}^{U} \bar{Q}_{i, L} \tilde{\phi}_{2} U_{j, R}+\xi_{i j}^{D} \bar{Q}_{i, L} \phi_{2} D_{j, R}+$ h.c.

where $\phi_{i}$, for $i=1,2$, are the two scalar doublets of a $2 \operatorname{HDM}\left(\tilde{\phi}_{i}=i \sigma^{2} \phi_{i}\right)$, while $\eta_{i j}^{U, D}$ and $\xi_{i j}^{U, D}$ are the non diagonal matrices of the Yukawa couplings.

In order to prevent FCNC to arise at the tree level, the scalar potential and Yukawa Lagrangian need to be constrained by an ad hoc discrete symmetry [5],

$$
\begin{array}{rll}
\phi_{1} \rightarrow-\phi_{1} & \text { and } & \phi_{2} \rightarrow \phi_{2} \\
D_{i} \rightarrow-D_{i} & \text { and } & U_{i} \rightarrow \mp U_{i} .
\end{array}
$$

Depending on whether the up-type and down-type quarks are coupled to the same or to two different scalar doublets respectively, one obtains the so called Model I and Model II 2HDM's. [14. 
In contrast we want to consider the case in which no discrete symmetry is imposed and both up-type and down-type quarks then have FC couplings. For this type of 2HDM, that we call Model III, the Yukawa Lagrangian for the quark fields is as in Eq. (11) and no term can be dropped a priori, see also Refs. [10, 12]. Since the two scalar doublet are completely independent, by a suitable rotation of the quark fields, we can chose the two scalar doublets in such a way that only the $\eta_{i j}^{U, D}$ couplings generate the fermion masses, i.e. such that

$$
<\phi_{1}>=\left(\begin{array}{c}
0 \\
v / \sqrt{2}
\end{array}\right), \quad<\phi_{2}>=0 .
$$

To the extent that the definition of the $\xi_{i j}^{U, D}$ couplings remains arbitrary, we will denote by $\xi_{i j}^{U, D}$ the new rotated couplings, such that the charged couplings look like $\xi^{U} \cdot V_{\mathrm{CKM}}$ and $V_{\mathrm{CKM}} \cdot \xi^{D}$. This form of the charged couplings is indeed peculiar to Model III compared to Models I and II and can have important phenomenological repercussions [16, 11].

The scalar physical mass spectrum consists of two charged $\phi^{ \pm}$and three neutral spin 0 bosons, two scalars $\left(H^{0}, h^{0}\right)$ and a pseudoscalar $\left(A^{0}\right)$,

$$
\begin{aligned}
H^{0} & =\sqrt{2}\left[\left(\operatorname{Re} \phi_{1}^{0}-v\right) \cos \alpha+\operatorname{Re} \phi_{2}^{0} \sin \alpha\right] \\
h^{0} & =\sqrt{2}\left[-\left(\operatorname{Re} \phi_{1}^{0}-v\right) \sin \alpha+\operatorname{Re} \phi_{2}^{0} \cos \alpha\right] \\
A^{0} & =\sqrt{2}\left(-\operatorname{Im} \phi_{2}^{0}\right),
\end{aligned}
$$

where $\alpha$ is a mixing phase which also determines the couplings between the neutral scalars and gauge bosons $\underbrace{2}\left(W^{ \pm}, Z^{0}\right)$, i.e.

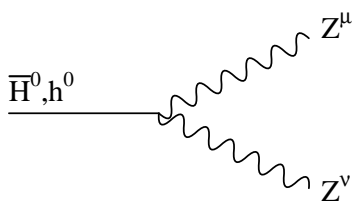

$$
\begin{aligned}
& \pm i \frac{g_{W}}{c_{W}} M_{Z}(\cos \alpha, \sin \alpha) g^{\mu \nu} \\
& \pm i g_{W} M_{W}(\cos \alpha, \sin \alpha) g^{\mu \nu} .
\end{aligned}
$$

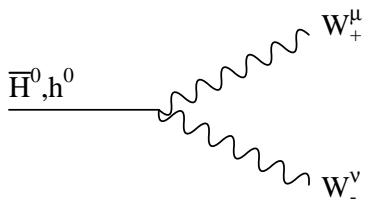

\footnotetext{
${ }^{2}$ We remind that in a $2 \mathrm{HDM}$ the pseudoscalar field $A^{0}$ does not couple to the gauge bosons.
} 
It is interesting to notice that for $\alpha=0$ : (1) $H^{0}$ corresponds exactly to the SM Higgs field, and $\phi^{ \pm}, h^{0}$ and $A^{0}$ generate the new FC couplings; (2) $h^{0}$ does not couple to the gauge bosons, i.e. it behaves like the pseudoscalar field $A^{0}$.

Finally, we want to introduce some definite ansatz that will guide us in our phenomenological approach to the study of Model III. Because the Yukawa Lagrangian directly breaks the flavor symmetry among quarks, and this ultimately results into fermion mass generation, some major proposals exist in the literature 6, 7, 8, 9] which suggest to take the new FC couplings to be proportional to the mass of the quarks involved in the coupling, i.e.

$$
\xi_{i j}=\lambda_{i j} \frac{\sqrt{m_{i} m_{j}}}{v},
$$

where for the sake of simplicity we take the $\lambda_{i j}$ to be real (for more details see [12, 11). In this ansatz the residual degree of arbitrariness of the FC couplings is expressed through the $\lambda_{i j}$ parameters, which need to be constrained by the available phenomenology. In particular we will see how $K^{0}-\bar{K}^{0}$ and $B^{0}-\bar{B}^{0}$ mixings (and to a less extent $D^{0}-\bar{D}^{0}$ mixing) put severe constraints on the FC couplings involving the first family of quarks.

There is no doubt that the most interesting signals of these non-standard couplings are to come from the physics of the top quark, both production and decays. Therefore, we would like to single out the right processes and the right environment in which we could already have the possibility of testing the consequences of our assumptions.

\section{Analysis of the constraints}

The existence of FC couplings is very much constrained by the experimental results on $F^{0}-\bar{F}^{0}$ flavor mixings (for $F=K, B$ and to a less extent $D$ )

$$
\begin{aligned}
\Delta M_{K} & \simeq 3.51 \cdot 10^{-15} \mathrm{GeV} \\
\Delta M_{B_{d}} & \simeq 3.26 \cdot 10^{-13} \mathrm{GeV} \\
\Delta M_{D} & <1.32 \cdot 10^{-13} \mathrm{GeV}
\end{aligned}
$$

due to the presence of new tree level contributions to each of the previous mixings. We have analyzed the problem in detail in Ref. [11], taking into account both tree level and loop contributions. Indeed the two classes of contributions can affect different FC couplings, due to the peculiar structure of the charged scalar couplings (see previous section).

We find that, unless for scalar masses in the multi-TeV range, the tree level contributions need to be strongly suppressed, requiring that the corresponding FC couplings are much than one. Enforcing the ansatz made in Eq. (5), this amounts to demand that 


$$
\lambda_{d s}^{D} \ll 1, \quad \lambda_{d b}^{D} \ll 1 \text { and } \lambda_{u d}^{U} \ll 1 .
$$

More generally, we can assume that the FC couplings involving the first generation are negligible. Particular 2HDM's have been proposed in the literature in which this pattern can be realized [15]. The remaining FC couplings, namely $\xi_{c t}^{U}$ and $\xi_{s b}^{D}$ are not so drastically affected by the $F^{0}-\bar{F}^{0}$ mixing phenomenology. From the analysis of the loop contributions to the $F^{0}-\bar{F}^{0}$ mixings (box and penguin diagrams involving the new scalar fields), we verify that many regions of the parameter space are compatible with the results in Eq. (6) [11]. Therefore we may want to look at other constraints in order to single out the most interesting scenarios.

Three are in particular the physical observables that impose strong bounds on the masses and couplings of Model III [16, 11]

- the inclusive branching ratio for $B \rightarrow X_{s} \gamma$, which is measured to be 17

$$
B R\left(B \rightarrow X_{s} \gamma\right)=(2.32 \pm 0.51 \pm 0.29 \pm 0.32) \times 10^{-4},
$$

- the ratio $R_{b}=\Gamma(Z \rightarrow b \bar{b}) / \Gamma(Z \rightarrow$ hadrons $)$, whose present measurement [18 is such that $R_{b}^{\text {expt }}>R_{b}^{\mathrm{SM}}(\sim 1.4 \sigma)$,

$$
R_{b}^{\text {expt }}=0.2170 \pm 0.0009 \text { while } R_{b}^{\mathrm{SM}}=0.2158,
$$

- the corrections to the $\rho$ parameter, which has become conventional to describe in terms of

$$
\rho_{0}=\frac{M_{W}^{2}}{\rho M_{Z}^{2} \cos ^{2} \theta_{W}}=1+\Delta \rho_{0}^{\mathrm{NEW}}
$$

where $\rho$ absorbs all the SM corrections to the gauge boson self energies and, in the presence of new physics, $\Delta \rho_{0}^{\mathrm{NEW}}$ summarizes the deviation from the SM prediction (i.e. $\rho_{0}=1$ ). From the recent global fits of the electroweak data, which include the input for $m_{t}$ from Ref. 19] and the new experimental results on $R_{b}, \rho_{0}$ turns out to be very close to unity. This imposes severe constraints on many extension of the SM, especially on the mass range of the new particles.

Since the experimental determination of $R_{b}$ is not completely definite, we demand less strict agreement between $R_{b}^{\exp }$ and $R_{b}^{\mathrm{SM}}$. In this case we find compatibility with the present experiments for

$$
\lambda_{c t} \simeq O(1) \text { and } \lambda_{s b} \simeq O(1) .
$$


The value of the mixing angle $\alpha$ is not determinant, while the masses are mainly dictated by the fit to $\operatorname{Br}\left(B \rightarrow X_{s} \gamma\right)$ and $\Delta \rho_{0}$ [16]. We are left with two possible scenarios,

$$
M_{H}, M_{h} \leq M_{c} \leq M_{A} \text { or } M_{A} \leq M_{c} \leq M_{H}, M_{h} .
$$

We conclude that, given the existing constraints, Model III has the very interesting characteristic of providing sizable FC couplings for the top quark, in a way that will certainly be testable at the next generation of lepton and hadron colliders. We will discuss some of these phenomenological issues in the next section.

\section{Top-charm production: the case of a muon col- lider}

If we assume Eq. (11), $\xi_{c t}^{U}$ becomes the most relevant FC coupling. The presence of a $\xi_{c t}^{U}$ flavor changing coupling can be tested by looking at both top decays and top production (see Ref. [11] and references therein).

It is interesting that the first upper bounds on $t \rightarrow \mathrm{cV}\left(V=\gamma, Z^{0}\right)$ are now coming from recent experimental analysis [20] (see Ref. [11] for the corresponding theoretical prediction in Model III). Encouraged by this progress, we want to concentrate here on top-charm production. The SM prediction for top-charm production is extremely suppressed and any signal would be a clear evidence of new physics with large FC couplings in the third family. The final state for this process has a unique kinematics, with a very massive jet recoiling against an almost massless one (very different from a $b s$ production signal, for instance). This quite distinctive signature may allow to work even with relatively low statistics, as can be the case for a lepton collider. The much better statistics one could get at an hadron collider, would come at a cost of a much higher background (mostly, tree level SM background for a one-loop process). A nice analysis of the hadron collider case is presented in Ref. [21].

Here we want to focus on lepton colliders and in particular on the case of the FMC. In principle, the production of top-charm pairs arises both at the tree level, via the $s$-channel exchange of a scalar field with FC couplings, and at the one loop level, via corrections to the $Z t c$ and $\gamma t c$ vertices.

The $s$-channel production is not relevant for an $e^{+} e^{-}$collider, because the coupling of the scalar fields to the electron is very suppressed $\left(m_{\mu} \simeq 200 m_{e}\right)$. An interesting proposal for a $t$-channel production via $W^{+} W^{-}$-fusion at a $\sqrt{s}=1$ $\mathrm{TeV} e^{+} e^{-}$collider has been pointed out in Ref. [22]. However, top-charm production at an $e^{+} e^{-}$collider remains mainly a loop effect and therefore (even at the energies of NLC) it is suppressed with respect to the corresponding production cross section at the FMC (see Ref. [12]) and in particular at the very high energy muon collider. 
In fact, for a $\mu^{+} \mu^{-}$collider, the $s$-channel top-charm production via a neutral scalar/pseudoscalar is a nice example of the kind of resonance production we briefly discussed in the Introduction. At resonance, the new scalars (let us denote them generically by $\mathcal{H}$ ) may be produced at an appreciable rate, and the effective cross section for any final state very much depends on the relation between the beam energy resolution and the width of the scalar particle $\left(\Gamma_{\mathcal{H}}\right)$ produced in the $s$-channel 23.

Following 23], we define the effective cross section as the convolution of the Breit-Wigner $\sigma_{t c}^{B W}$ cross section with a gaussian beam energy spread,

$$
\sigma_{t c}^{e f f}=\int d \sqrt{s^{\prime}} \frac{\exp \left[-\left(\sqrt{s^{\prime}}-\sqrt{s}\right)^{2} / 2 \sigma^{2}\right]}{\sqrt{2 \pi} \sigma} \sigma_{t c}^{B W}\left(s^{\prime}\right)
$$

where the rsm of the gaussian distribution is defined in terms of the resolution parameter $R$ as

$$
\sigma=7 \mathrm{MeV}\left(\frac{R}{0.01}\right)\left(\frac{\sqrt{s}}{100 \mathrm{GeV}}\right) .
$$

If $\sigma \gg \Gamma_{H}$ the effective cross section is suppressed as $\Gamma_{\mathcal{H}} / s$,

$$
\sigma_{t c}^{e f f}=\frac{\pi \Gamma_{H}}{2 \sqrt{2 \pi} \sigma} \sigma_{B W}\left(s=M_{\mathcal{H}}\right),
$$

while the optimal case is reached if $\sigma \ll \Gamma_{H}$, when

$$
\sigma_{t c}^{e f f}=\sigma_{B W}\left(s=M_{\mathcal{H}}\right)
$$

with a whole spectrum of possible intermediate cases. In our analysis we study

$$
R_{t c}=\frac{\sigma_{t c}^{e f f}}{\sigma_{0}}=R(\mathcal{H})(B(\mathcal{H} \rightarrow \bar{t} c)+B(\mathcal{H} \rightarrow \bar{c} t))
$$

where $\sigma_{0}=\sigma\left(\mu^{+} \mu^{-} \rightarrow \gamma \rightarrow e^{+} e^{-}\right)$and $R(\mathcal{H})=\sigma_{\mathcal{H}} / \sigma_{0}$ for $\sigma_{\mathcal{H}}$ the total cross section for producing $\mathcal{H}$.

To be more explicit, let us consider the case of a scalar field $\mathcal{H}=h^{0}$. Then, according to what we discussed in a previous section,

$$
C_{h^{0} t c}=\frac{1}{\sqrt{2}}\left[\xi_{t c} P_{R}+\xi_{c t}^{\dagger} P_{L}\right] \cos \alpha \equiv \frac{g \sqrt{m_{t} m_{c}}}{2 M_{W}}\left(\chi_{R} P_{R}+\chi_{L} P_{L}\right)
$$

The total width $\Gamma_{h^{0}}$ can be obtained from the literature (see for instance [14), and varies with $M_{h^{0}}$ because of the different decay channels that open up at higher $M_{h^{0}}$. On the other hand, the rate for top-charm production in Model III is explicitly given by,

$$
\Gamma\left(h^{0} \rightarrow t \bar{c}\right)=\frac{3 g^{2} m_{t} m_{c} M_{h^{0}}}{32 \pi M_{W}^{2}}\left(\frac{\left(M_{h^{0}}^{2}-m_{t}^{2}\right)^{2}}{M_{h^{0}}^{4}}\right)\left(\frac{\left|\chi_{R}\right|^{2}+\left|\chi_{L}\right|^{2}}{2}\right)
$$


Therefore, our results depend on both $M_{h^{0}}$ and $\Gamma_{h^{0}}$, and on how $\Gamma_{h^{0}}$ compares to the resolution parameter $R$. We use the set of parameters posted for this workshop. In particular we consider the possibility to reach resolutions as accurate as $R \simeq 1 \%$ and the availability of a average luminosity of $\mathcal{L}_{\mathrm{av}}=10^{32}$ for $\sqrt{s}=200 \mathrm{GeV}$ up to $\mathcal{L}_{\mathrm{av}}=7 \times 10^{32}$ for $\sqrt{s}=500 \mathrm{GeV}$.

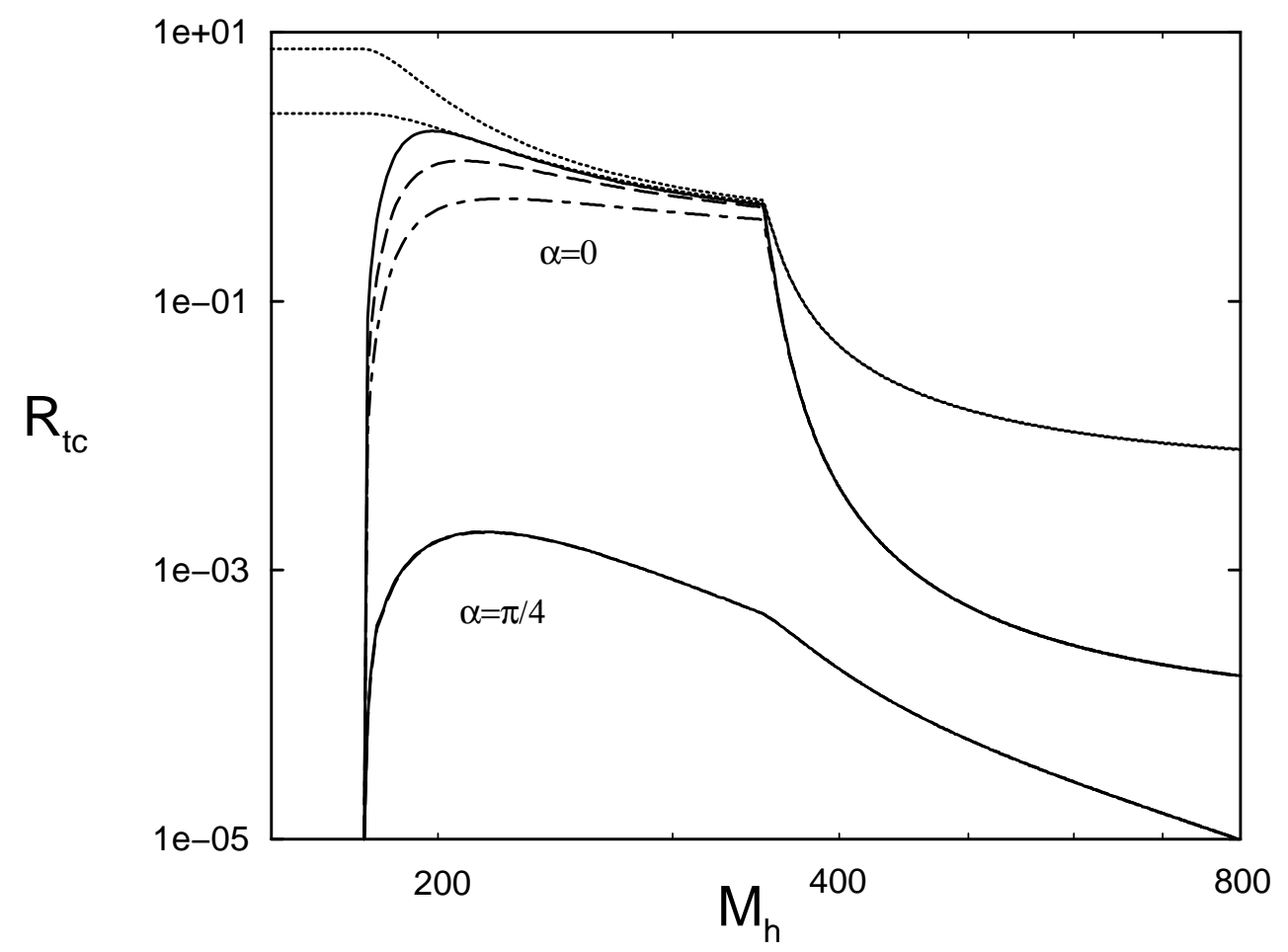

Figure 1: The value of $R\left(h^{0}\right)$ is shown as a function of $M_{h^{0}}$ in a pure BreitWigner case (upper dotted line) and when the gaussian width distribution of the beam energy (for $R=0.01$ ) is taken into account (lower dotted line). The ratio $R_{t c}$ is also shown for different values of the resolution parameter $R=0.001$ (solid), 0.01 (dashed) and 0.03 (dot-dashed), when $\alpha=0$ (upper group of curves) and when $\alpha=\pi / 4$ (lower group of curves).

For a given $R$, we vary $M_{h^{0}}$ in the range,

$$
100 \mathrm{GeV} \leq M_{h^{0}} \leq 800 \mathrm{GeV},
$$

and for given $M_{h^{0}}$ and $R$, we consider two different cases: $\alpha=0$ and $\alpha=\pi / 4$. In the first case $\Gamma_{h^{0}}$ is smaller than in the second case, because the $h^{0}$ field does 
not couple to $W^{+} W^{-}$and $Z^{0} Z^{0}$. In both cases we assume all the $\chi_{i}$ couplings to be real and of $O(1)$. Our results are summarized in Fig. 1.

Note that for $\alpha=0$, if $M_{h^{0}}$ is below the $t \bar{t}$ threshold $R_{t c}$ is about $.2-3$ and in fact $t c$ makes up a large branching ratio. Above the $t \bar{t}$ threshold $R_{t c}$ drops. In this narrow width case we clearly see how our result depends on $R$ and how for smaller values of $R$ the predictions get closer and closer to the pure BreitWigner case. For $\alpha=\pi / 4$ the branching ratio is smaller due to the $W^{+} W^{-}$and $Z^{0} Z^{0}$ threshold at about the same mass as the $t c$ threshold and so $R_{t c}$ is around $10^{-3}$. In this case the width is broader and there is almost no dependence on $R$. To be more specific, let us assume that $M_{h^{0}}=300 \mathrm{GeV}$, then $\sigma_{0} \approx 1 \mathrm{pb}$. For $\mathcal{L}_{\mathrm{av}}=10^{32} \mathrm{~cm}^{-2} \mathrm{~s}^{-1}$ and $R=0.01, \alpha=0$ will produce about $10^{2}(t \bar{c}+\bar{t} c)$ events and $\alpha=\pi / 4$ will produce only a few events. Much higher statistics can be obtained improving on the average luminosity available, which we hope will remain one of the priorities in the study of the FMC. Given the distinctive nature of the final state and the lack of a Standard Model background, sufficient luminosity should allow the observation of such events.

If such events are observed, we will also have the possibility to extract the values of $\chi_{L}$ and $\chi_{R}$, provided we determine the helicity of the produced top quark, expressed in termes of $\chi_{L}$ and $\chi_{R}$ as

$$
\mathbf{H}_{t}=-\mathbf{H}_{\bar{t}}=\frac{\left|\chi_{R}\right|^{2}-\left|\chi_{L}\right|^{2}}{\left|\chi_{R}\right|^{2}+\left|\chi_{L}\right|^{2}}
$$

The helicity of the $t$ quark cannot be determined directly, but has to be obtained from the decay distributions of the top [24, the number of events required to observe it with a significance of $3 \sigma$ being

$$
N_{3 \sigma}=\frac{36}{\mathcal{E}_{t}^{2} \mathbf{H}_{t}^{2}} \approx \frac{107}{\mathbf{H}_{t}^{2}} .
$$

Thus at least $10^{2}$ events are required to begin to measure the helicity of the top and hence the relative strengths of $\chi_{L}$ and $\chi_{R}$. In the above numerical examples it is clear that for some combinations of parameters, particularly if the luminosity is higher than $10^{32} \mathrm{~cm}^{-2} \mathrm{~s}^{-1}$, sufficient events to measure the helicity may be present.

\section{Acknowlegement}

This research was supported by the U.S. Department of Energy under Contract DE-FG02-95ER40896.

\section{References}

[1] V. Barger, this proceedings. 
[2] J. Gunion, this proceedings.

[3] T. Han and M. Demarteau, this proceedings.

[4] M. Carena and S. Protopopescu, this proceedings.

[5] S.L. Glashow and S. Weinberg, Phys. Rev. D15, 1958 (1977).

[6] T.P. Cheng and M. Sher, Phys. Rev. D35, 3484 (1987).

[7] M. Sher and Y. Yuan, Phys. Rev.D44, 1461 (1991).

[8] A. Antaramian, L.J. Hall, and A. Rasin, Phys. Lett. B69, 1871 (1992).

[9] L.J. Hall and S. Weinberg, Phys. Rev. D48, R979 (1993).

[10] M. Luke and M.J. Savage, Phys. Lett. B307, 387 (1993).

[11] D. Atwood, L. Reina and A. Soni, Phys. Rev. D55, 3156 (1997).

[12] D. Atwood, L. Reina and A. Soni, Phys. Rev. D53, 1199 (1996).

[13] D. Atwood, L. Reina and A. Soni, Phys. Rev. Lett. 75, 3800 (1995).

[14] For a review see J. Gunion, H. Haber, G. Kane, and S. Dawson, The Higgs Hunter's Guide, Addison-Wesley, New York, 1990.

[15] A. Das and C. Kao, Phys. Lett. B372, 106 (1996).

[16] D. Atwood, L. Reina and A. Soni, Phys. Rev. D54, 3295 (1996).

[17] R. Ammar et al., CLEO Collaboration, Phys. Rev. Lett. 71, 674 (1993); M.S. Alam et al., CLEO Collaboration, Phys. Rev. Lett. 74, 2885 (1995).

[18] The LEP Electroweak Working group, LEPEWWG/97-02.

[19] P. Giromini, Proccedings of the LP'97, Hamburg, 1997; A. Yagil, Proceedings of the International Europhysics Conference, Jerusalem, 1997.

[20] F. Abe et al., CDF Collaboration, FERMILAB-PUB-97-270; T. J. LeCompte, CDF Collaboration, Proceedings of the Symposium on FCNC, Santa Barbara, 1997.

[21] T. Han, R.D. Peccei and X. Zhang, Nucl. Phys. B454, 527 (1995).

[22] S. Bar-Shalom, G. Eilam, A. Soni, J. Wudka, Phys. Rev. Lett. 79, 1217 (1997); hep-ph/9708358.

[23] V. Barger, M.S. Berger, J.F. Gunion and T. Han, Phys. Rev. Lett. 75, 1462 (1995); Phys. Rept. 286, 1 (1997).

[24] D. Atwood and A. Soni, Phys. Rev. D52, 6271 (1995). 